\title{
Mapping of QTL for total spikelet number per spike on chromosome 2D in wheat using a high-density genetic map
}

\author{
Mei Deng ${ }^{2 *}$, Fangkun $\mathrm{Wu}^{2 *}$,Wanlin $\mathrm{Zhou}^{2}$, Jing $\mathrm{Li}^{2}$, Haoran $\mathrm{Shi}^{2}$, Zhiqiang Wang ${ }^{2}$, Yu Lin ${ }^{2}$, Xilan Yang ${ }^{2}$, \\ Yuming Wei ${ }^{1,2}$, Youliang Zheng ${ }^{1,2}$ and Yaxi Liu ${ }^{1,2}$ iD \\ ${ }^{1}$ State Key Laboratory of Crop Gene Exploration and Utilization in Southwest China, Wenjiang, Chengdu \\ 611130, China. \\ ${ }^{2}$ Triticeae Research Institute, Sichuan Agricultural University, Wenjiang, Chengdu 611130, China.
}

\begin{abstract}
Total spikelet number per spike (TSS) is one of the key components of grain yield in wheat. Chromosome (chr.) 2D contains numerous genes that control TSS. In this study, we evaluated $138 \mathrm{~F}_{8}$ recombinant inbred lines (RILs) derived from an $\mathrm{F}_{2}$ population of a synthetic hexaploid wheat line (SHW-L1) and a common wheat cultivar (Chuanmai 32) for TSS in six different environments. To identify quantitative trait loci (QTL) for TSS, we constructed an integrated high-density genetic map of chr. 2D containing two simple sequence repeats, 35 diversity array technology markers, and 143 single nucleotide polymorphisms. We identified three stable QTL for TSS that individually explained $9.7-19.2 \%$ of the phenotypic variation and predicted 23 putative candidate genes within the QTL mapping interval. Overall, our results provide insight into the genetic basis of TSS in synthetic hexaploid wheat that may be useful in breeding high-yielding wheat cultivars.
\end{abstract}

Keywords: recombinant inbred line, synthetic hexaploid wheat, quantitative trait locus, total spikelet number per spike.

Received: May 09, 2018; Accepted: December 4, 2018.

\section{Introduction}

To feed the ever-growing population, improving the yield of wheat, one of the most important food crops globally, is becoming increasingly important (Rajaram, 2001; Godfray et al., 2010; Reynolds et al., 1996). Among the factors determining wheat yield, total spikelet number per spike (TSS) is considered one of the key factors, and previous studies have shown that spikelet number is closely related to grain number (Rawson, 1970) and determines where spikelets can set (Slafer and Andrade, 1993). As the basal units of inflorescences, spikelets are crucial for reproductive success and final yield (Cai et al., 2014).

TSS, as an important quantitative agronomic trait, is controlled by polygenes and influenced by the environment (Zhou et al., 2017). Understanding the genetic factors underlying variations in TSS without environmental interference is essential for the genetic improvement of wheat (Mackay, 2001; Wurschum, 2012). Previous genetic studies have revealed that chromosome (chr.) 2D is rich in genes that control spikelet number per spike in common wheat, and many quantitative trait loci (QTL), such as QSsn.cau-2D.2 and QSpn.nau-2D, have been discovered

Send correspondence to Yaxi Liu. Triticeae Research Institute, Sichuan Agricultural University, Wenjiang, 611130 Chengdu, China. E-mail: liuyaxi@ sicau.edu.cn; yaxi.liu@ foxmail.com.

${ }^{*}$ These authors contributed equally to this work. on this chromosome (Ma et al., 2007; Zhai et al., 2016). However, information about TSS-QTL on chr. 2D is still limited for synthetic hexaploid wheat (SHW), which contains a combination of genes from Aegilops tauschii and common wheat (Triticum aestivum), as well as novel functional genes (Mares and Mrva, 2008). Yu et al. (2014) identified a stable QTL (in the region wPt-6133-gpw4473) for TSS on chr. 2D in a population developed from a cross between SHW (SHW-L1) and the common wheat variety Chuanmai 32, using a genetic map containing simple sequence repeats (SSRs) and diversity arrays technology (DArT) markers. To accurately parse this QTL, we integrated the markers reported by Yu et al. (2014) with novel SNP markers into a new chr. 2D high-density genetic map and identified QTL for TSS. Our data might help to better understand the genetic basis of TSS in SHW and accelerate the development of new high-yielding wheat cultivars.

\section{Materials and Methods}

\section{Plant material}

A total of $138 \mathrm{~F}_{8}$ recombinant inbred lines (RILs) derived from an $\mathrm{F}_{2}$ SHW-L1/Chuanmai 32 population were used to construct an integrated linkage map for chr. 2D and detect QTL for TSS. SHW-L1 is an SHW derived from a cross between T. turgidum ssp. turgidum AS2255 (AABB) 
and A. tauschii ssp. tauschii AS60 (DD) (Zhang et al., 2004), whereas Chuanmai 32 is a commercial hexaploid wheat cultivar grown in the southwest winter-wheat areas of China. Transgressing segregations for TSS have been previously observed in SHW-L1/Chuanmai RILs, and a total of 68 SSRs and 1794 DArT markers for important agronomic traits have been mapped (Yu et al., 2014).

\section{Field experiment and phenotyping}

All RILs and their parents were evaluated in a completely randomized block design with two replicates, at the experimental stations of Dujiang Weir $\left(31^{\circ} 01^{\prime} \mathrm{N}\right.$ and $103^{\circ} 32^{\prime} \mathrm{W}$ ) in 2008, 2009, and 2010 (environments E1, E2, and E3, respectively), Guanghan $\left(30^{\circ} 99^{\prime} \mathrm{N}\right.$ and $\left.104^{\circ} 25^{\prime} \mathrm{W}\right)$ in 2009 and 2010 (environments E4 and E5), and Wenjiang (30 $36^{\prime} \mathrm{N}$ and $103^{\circ} 41^{\prime} \mathrm{W}$ ) in 2011 (environment E6). Plants were sown in single $1.5-\mathrm{m}$ rows with a $30-\mathrm{cm}$ space between rows and a $10-\mathrm{cm}$ space between individuals. Data for TSS were manually counted from 10 randomly selected guarded main spikes from each line in each environment (Yu et al., 2014).

\section{Statistical analysis}

To estimate random effects, a best linear unbiased prediction (BLUP) mixed model was used to obtain BLUP-TSS values (Piepho et al., 2008). The BLUP for the phenotypic value of plant $Y_{i}$ was calculated as follows: $Y_{i}=$ $X_{i} f+a_{i}+e_{i}$, where $f$ is a vector of fixed effects, $X_{i}$ is an incidence vector, $e_{i}$ is the environmental deviation, and $a_{i}$ is the phenotypic value (Goddard, 1992). An analysis of variance (ANOVA) was performed using SAS 9.1.3 (SAS Institute, Cary, NC, USA) to estimate the effects of genotype on TSS. The estimated broad-sense heritability of TSS was calculated as follows: $h=\sigma^{2} \mathrm{G} /\left(\sigma^{2} \mathrm{G}+\sigma^{2} \mathrm{e} / \mathrm{r}\right)$, where $\sigma^{2} \mathrm{G}$ is the genetic variance, $\sigma^{2}$ e is the residual variance, and $r$ is the number of replicates per genotype.

\section{Construction of a genetic map for chr. 2D}

A total of 13 SSRs, 93 DArT markers, and 2306 SNPs reported in previous studies (Yu et al., 2014; Yang, 2016) were used to construct a genetic map for chr. 2D. After the removal of redundant markers that were located on the same loci (Yang, 2016), the genetic map consisted of 13 SSRs, 86 DArT markers, and 244 SNPs. The remaining markers were assigned to linkage groups using Joinmap 4.0 (Van Ooijen, 2006) with a recombination frequency of $0.25-0.05$. The final genetic distances were obtained using the Kosambi mapping function (Kosambi, 2016).

\section{QTL mapping}

QTL screening was conducted using interval mapping (IM) in MapQTL 6.0 (Van Ooijen, 2009). Logarithm of odds (LOD) threshold values for IM were determined based on 1000 permutations to declare significant QTL at $p<0.05$, whereas QTL with LOD values $<3.0$ were excluded to ensure the authenticity and reliability of the reported QTL. QTL that explained more than $10 \%$ of variation in TSS were considered as major QTL.

\section{Prediction of candidate genes}

To predict candidate/flanking genes, the interval flanking marker sequence was aligned via a BLAST search against the International Wheat Genome Sequencing Consortium and EnsemblPlants databases to determine the position with the highest identity and detect genes within the closed interval. To predict the function of the candidate genes, we conducted Gene Ontology (GO) annotation and Kyoto Encyclopedia of Genes and Genomes (KEGG) pathway enrichment analysis at $p<0.05$, using Arabidopsis thaliana as a background species, in KOBAS 3.0.

\section{Results}

\section{TSS variation in RILS}

The results of the mean phenotypic performance and BLUP values for the TSS of RILs and their parents in the six environments are presented in Table 1. The ANOVA and heritability $\left(h^{2}\right)$ values are presented in Table 2 . Variation among the RILs was high, with a coefficient of variation ranging from $9.53 \%$ in E1 to $14.39 \%$ in E6. Distributions were continuous across all environments (Figure 1), and, thus, the RILs were suitable for analyzing QTL for TSS.

Table 1 - The mean phenotypic performance for TSS of the recombinant inbred lines (RILs) and their parents in six environments.

\begin{tabular}{|c|c|c|c|c|c|c|c|}
\hline \multirow[t]{2}{*}{ Environments } & \multicolumn{2}{|c|}{ Parent } & \multicolumn{5}{|c|}{ Population } \\
\hline & SHW-L1 & Chuanmai 32 & Min & Max & Mean & SD & CV (\%) \\
\hline E1 & - & - & 16.80 & 27.20 & 22.12 & 2.11 & 9.53 \\
\hline $\mathrm{E} 2$ & 20.4 & 21 & 15.00 & 26.40 & 20.27 & 2.28 & 11.24 \\
\hline E3 & 21.6 & 20.5 & 13.60 & 27.20 & 19.86 & 2.40 & 12.07 \\
\hline E4 & 20.8 & 25 & 12.71 & 24.00 & 18.31 & 2.35 & 12.85 \\
\hline E5 & 21.2 & 22.4 & 14.00 & 28.00 & 19.36 & 2.63 & 13.57 \\
\hline E6 & 19.6 & 19.6 & 13.33 & 26.20 & 18.67 & 2.69 & 14.39 \\
\hline BLUP & 20.9 & 21.8 & 15.49 & 23.85 & 19.78 & 1.67 & 8.46 \\
\hline
\end{tabular}



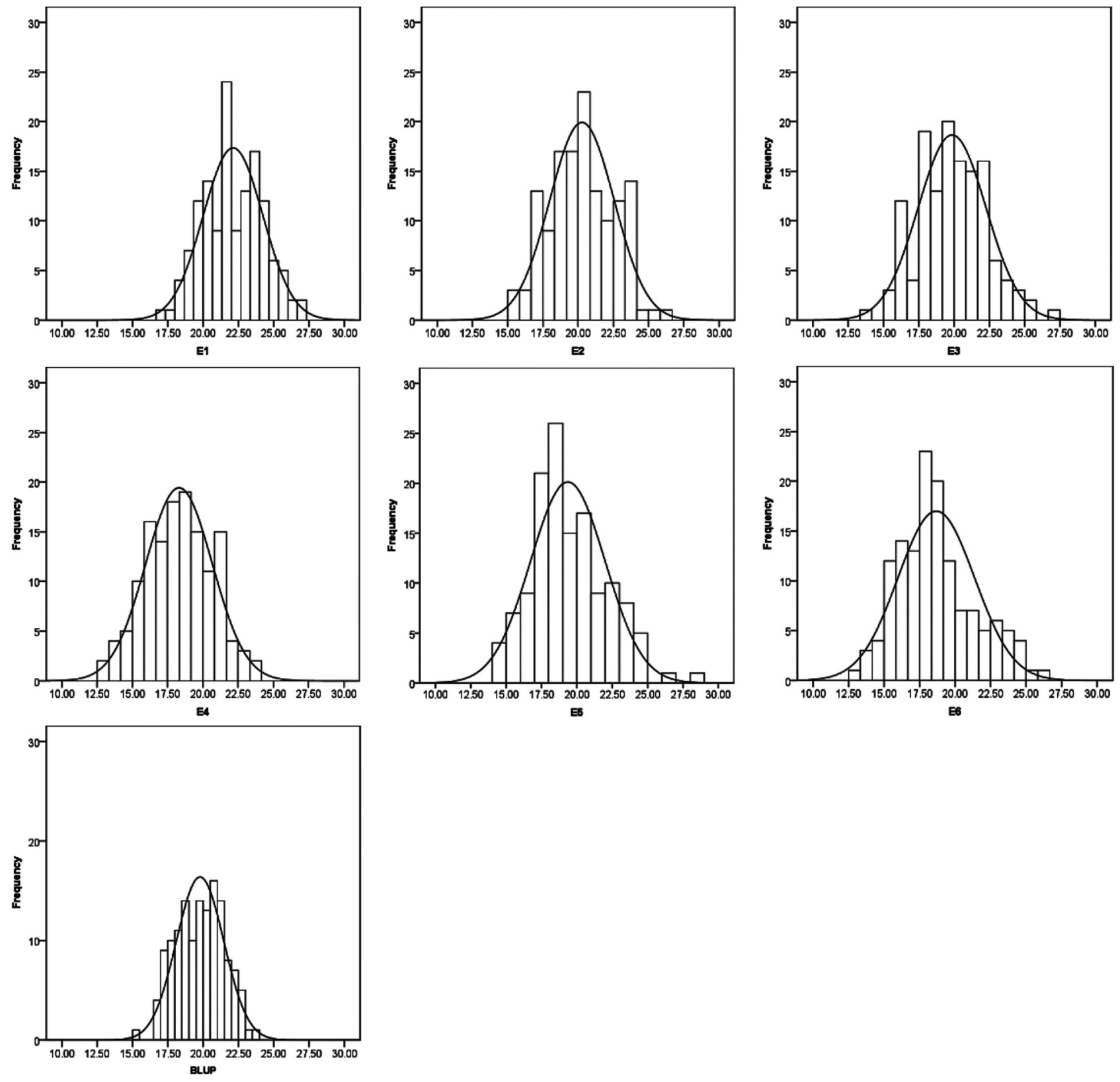

Figure 1 - Frequency distribution of TSS in the SHW-L1/Chuanmai 32 recombinant inbred line (RIL) population under 6 environments. The horizontal axis indicates TSS value, the ordinate axis indicate frequency.

\section{Construction of genetic linkage map for chr. 2D}

Different types of molecular markers were used to construct a genetic map for chr. 2D. At a maximum recombination frequency score of 0.4 and a minimum LOD score of 1.00, 180 markers were assigned to two different linkage groups (LG) that covered $207.33 \mathrm{cM}$, with a mean interval distance of $1.15 \mathrm{cM}$ between the markers; however, the other 163 markers remained unassigned. LG 1 consisted of two SSRs, 35 DArT markers, and 90 SNPs, whereas LG 2 consisted of 53 SNPs.

\section{Stable QTL for TSS}

Three QTL for TSS (QTSS.sicau-2D.1, QTSS.sicau2D.2, and QTSS.sicau-2D.3) with significant effects in at least four environments were identified on chr. 2D. The existence and stability of the QTL were confirmed by BLUP values. The three major QTL individually explained 9.7-19.2\% of the phenotypic variation (Table 3, Figure 2), and the additive effects of QTL showed that the positive alleles (i.e., those related to a high number of TSS) on chr. 2D originated from SHW-L1. Of these, QTSS.sicau-2D.1 was detected in all environments, except E5, whereas 


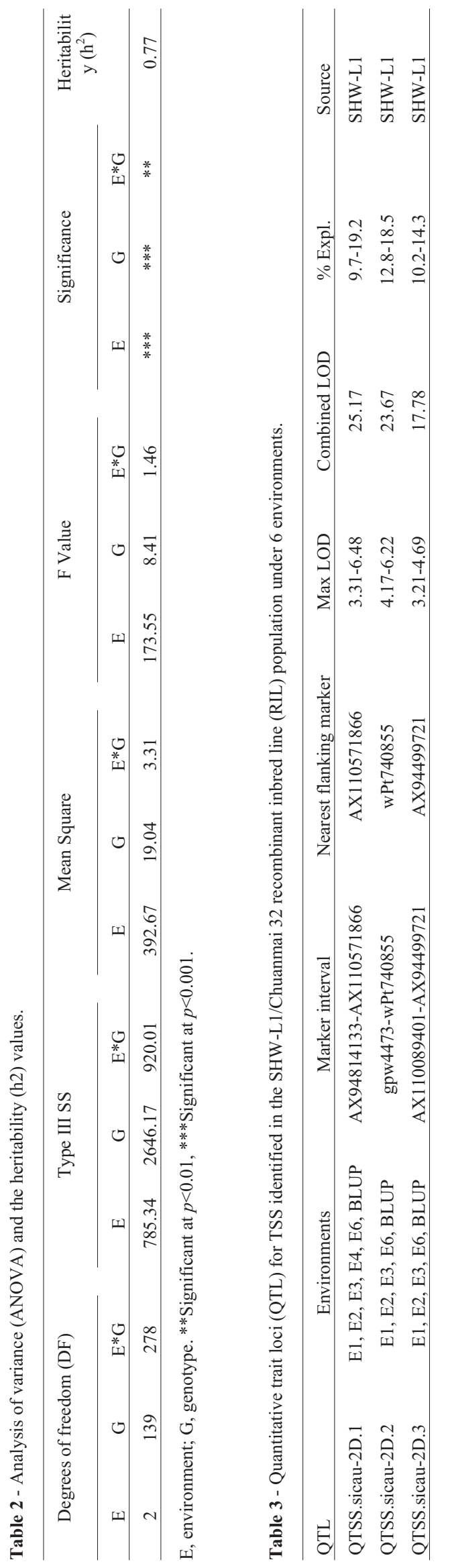

Chromesome 2D

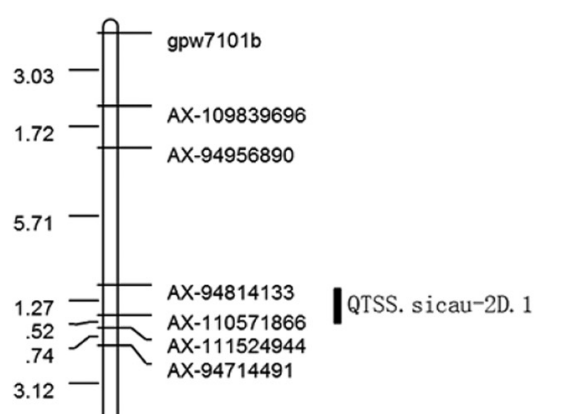

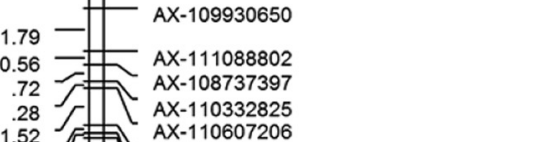

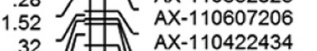

.32 AX-110422434

$.19]\left[\begin{array}{l}A X-111469300 \\ -19\end{array}\right.$

$\left.\begin{array}{l}.9 \\ 85\end{array}\right][7]\left[\begin{array}{l}A X-111409300 \\ A X-111482016\end{array}\right.$

$.84]\left[\begin{array}{l}\text { AX-110459643 } \\ .84-110348073\end{array}\right.$

$.86]\left[\begin{array}{l}A X-110459643 \\ A X-110348073\end{array}\right.$

$1.35]\left[\begin{array}{l}\mathrm{AX}-111028331 \\ \mathrm{AX}-1.667978\end{array}\right.$

$\left.\begin{array}{r}.02 \\ 1.65\end{array}\right]$ [

$\left.\begin{array}{c}1.55 \\ .47\end{array}\right]\left[\begin{array}{l}.6 \\ A X-94410456 \\ \text { AX- } 94848004\end{array}\right.$

$\left.\begin{array}{l}.47 \\ .04\end{array}\right]-\left[\begin{array}{l}A X-944848004 \\ W P t-666223\end{array}\right.$

$\left.\begin{array}{r}.04 \\ 3.06\end{array}\right]$ [ WPt-9997

$1.04]-\left[\begin{array}{l}\text { wPw } \\ \text { gpw73 } \\ \text { wPt-740855 }\end{array}\right.$

.06 .

$.02] .=\left[\begin{array}{l}\text { wPt-6609 } \\ \text { wPt-665328 }\end{array}\right.$

$.01]$ wPt-741029

$.06]\left[\begin{array}{l}w P t-731589 \\ w P t-730529\end{array}\right.$

$\left.\begin{array}{r}.01 \\ 1.48\end{array}\right]$ |

2.29 wPt-730868

$.33]\left[\begin{array}{ll}\mathrm{AX}-94665408 \\ -0.78-667843\end{array}\right.$

.08 .

$\left.\begin{array}{r}4.45 \\ 41\end{array}\right]\left[\begin{array}{l}\text { wPt-666857 } \\ \text { AX-109914934 }\end{array}\right.$

.41
1.33

$\left.\begin{array}{l}1.33 \\ 2.75\end{array}\right]$ ED AX-110484736

$\left.\begin{array}{l}2.75 \\ 1.08\end{array}\right]$ AX-108730977

.34 AX-109356942

$.53][$ AX-109828522

$.11]$ AX-111787449

$.02]-\begin{aligned} & A X-94576263 \\ & \text { AX-111730196 }\end{aligned}$

.31 AXt-9848

.04
.32

.12 AX-94803794

.14

$.15]-\left[\begin{array}{l}A X-109454707 \\ A X-108923452\end{array}\right.$

$\left.\begin{array}{l}.44 \\ 15\end{array}\right]=\begin{aligned} & A X-108923452 \\ & A X-95230029\end{aligned}$

.21 AX-94959151

.09 - $A X-110637076$

$1.69-\left[\begin{array}{l}A X-110474313 \\ A X-110072750\end{array}\right.$

$.27] \quad \begin{aligned} & A X-110072750 \\ & A X-111297034\end{aligned}$

$.41]-\left[\begin{array}{l}A X-110617078 \\ -3\end{array}\right]$

$\left.\begin{array}{r}1.12 \\ .34\end{array}\right] \quad \begin{aligned} & A X-110089401 \\ & A X-94499721\end{aligned}$

| QTSS. sicau-2D. 3

| QTSS. sicau-2D. 2

3 - $A X-108984683$

AX-108987045

1.11 - AX-108989332

$.15 \|\left[\begin{array}{l}A X-109899332 \\ \text { wPt-665645 }\end{array}\right.$

$.03]\left[\begin{array}{l}A X-111181506 \\ A X-111606890 \\ A X-111057231\end{array}\right.$

$\left.\begin{array}{l}1.01 \\ .57\end{array}\right]$ [ $\begin{aligned} & A X-111057231 \\ & A X-110514436 \\ & A X-111017517 \\ & A X-109581436\end{aligned}$

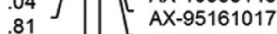

5.96

AX-94919637

AX-111347722

Figure 2 - Chromosomal locations of quantitative trait loci for TSS and associated markers in the SHW-L1/Chuanmai 32 recombinant inbred line (RIL) population under 6 environments. The black bar points to the LOD peak of QTL. 
QTSS.sicau-2D.2 and QTSS.sicau-2D.3 were found in four environments (E1-3 and E6).

\section{Putative candidate genes in QTL intervals}

A total of 23 putative candidate genes associated with TSS were identified (Table S1). Two genes were predicted in the QTSS.sicau-2D.1 interval (IWGSC_ref_V1_chr2D chr2D:9346330-9579108), 19 in the QTSS.sicau-2D.2 interval (IWGSC_ref_V1_chr2D chr2D:3822275443976070), and two in the QTSS.sicau-2D.3 interval (IWGSC ref V1 chr2D chr2D:77381440-78089285). Of these, five genes, LECRK42, AT2G34930, PME21, $C O B L 7$, and $P I P 5 K 4$, regulate flower development, and three, $C R K 8, R P P L 1$, and $A T 4 G 29780$, are related to spikelet number differentiation. KEGG pathway enrichment analysis showed that PIP5K4 is involved in inositol phosphate metabolism, phosphatidylinositol signaling system, and endocytosis; PME21 and PME53 are involved in starch and sucrose metabolism and pentose and glucoronate interconversions; and AT2G07689 and ATP1are involved in oxidative phosphorylation.

\section{Discussion}

In the present study, by using an integrated highdensity genetic map, three major QTL for TSS were detected on chr. 2DS (short arm of chr. 2D). Among them, QTSS.sicau-2D.2 was located in the marker interval wPt6133-gpw4473, which might correspond to that reported by Yu et al. (2014) in the same marker interval (Figure 3). Notably, using the integrated high-density genetic map for chr. 2D, we managed to decrease the marker interval range from $15.6 \mathrm{cM}$ to $1.04 \mathrm{cM}$, which is a substantial improvement over that obtained in previous studies, and two additional QTL were detected. Similarly, by highdensity consensus map, Marone et al. (2012) increased the map density from $11.8 \mathrm{cM}$ per marker (as obtained by Nachit et al., 2001) to $1.6 \mathrm{cM}$ per marker, and Sourdille et al. (2003) confirmed previously detected QTL and identified three novel ones, suggesting that good coverage of chromosome is important for QTL detection. Therefore, this study provides a strategy for identifying QTL, which combines new molecular data with phenotypic data and enables possible detection of previously overlooked QTL.

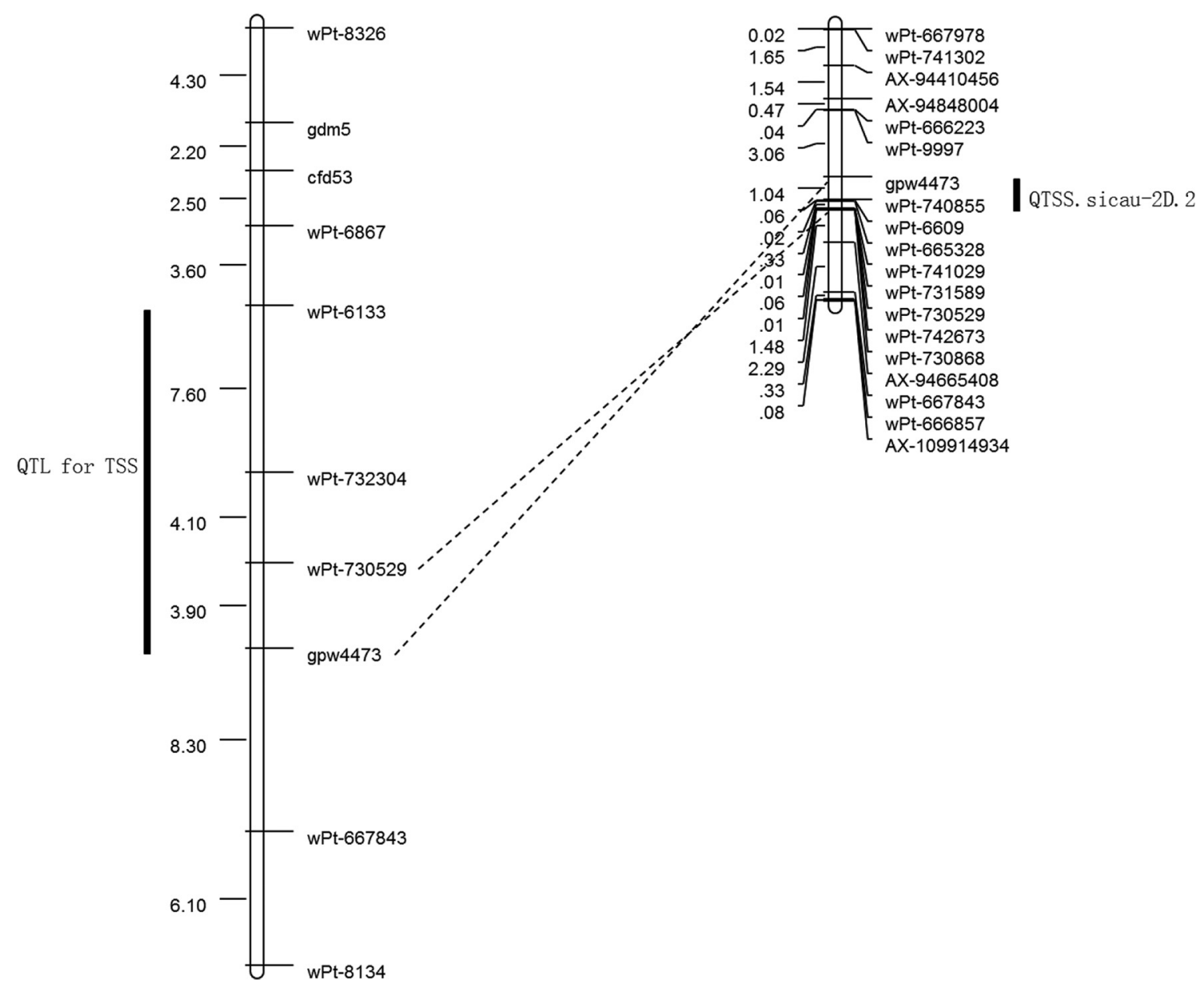

Figure 3 - A comparison of stable putative QTL for TSS between a former study (Yu et al., 2014) and our result. The left side shows the results of previous studies, and the right side shows the results of our studies. 
For chr. 2D, previous studies have identified numerous putative QTL (Li et al., 2002; Sourdille et al., 2003; Quarrie et al., 2006; Cui et al., 2012; Liu et al., 2014; Zhai et al., 2016; Zhou et al., 2017). Ma et al. (2007) reported two QTL for TSS in the marker intervals Xwmc181.1Xaf12d (near IWGSC_ref_V1_chr2D chr2D:593738612593738636) and $-\bar{X}$ af12-Xcfd239 (near IWGSC_ref_V1_chr2D chr2D: 647432804-647432824); Cui et $\overline{a l} .(\overline{2012)}$ also reported two QTL for TSS in the marker intervals Xcfd267-Xmag3596 (near IWGSC_ref V1_chr2D chr2D:608198901-608198921) and Xbarc228-Xwmc181.1 (near IWGSC_ref_V1_chr2D chr2D:593738612-593738636). Zhou et al. (2017) also reported a QTL for TSS, named QTsn.czm-2D.3 (near IWGSC_ref_V1_chr2D chr2D:480324893-480325330). Comparison with data in the IWGSC database revealed that these above-mentioned QTL were found on chr.2DL (long arm of chr.2D). The three QTL we detected were located on chr. 2DS; so, we paid more attention to the QTL previously detected on chr. 2DS. Li et al. (2002) reported a QTL for TSS in the marker interval Xbcd611-Xgwm484 (IWGSC_ref_V1_chr2D chr2D: 34894502-48174395) on chr. 2DS; by comparison, this marker interval is different from those of QTSS.sicau-2D.1 (IWGSC_ref_V1_chr2D chr2D:9346330-9579108) and QTSS.sicau-2D.3 (IWGSC_ref_V1_chr2D chr2D:77381440-78089285), but contains $\bar{Q} T S \bar{S}_{\text {sicau-2D.2 }}$ (IWGSC_ref_V1_chr2D chr2D:38222754-43976070). Zhou et al. (2017) also reported two QTL for TSS on chr. 2DS: QTsn.czm-2D.2 was located in the marker interval XPpd_D12DS_5382880_5243 (IWGSC_ref_V1_chr2D chr2D:29716047-67557838) that contains QTSS.sicau2D.2, while the other QTL, named QTsn.czm-2D.1 (IWGSC ref V1 chr2D chr2D:19623154-29716165), was different from QTSS.sicau-2D.1 and QTSS.sicau2D.3. Therefore, QTSS.sicau-2D.1 and QTSS.sicau-2D.3 are probably novel QTL that can be used for further fine mapping and genetic analysis.

In wheat, the development of polymorphism markers based on QTL is an effective method for molecular-assisted breeding (Roussel et al., 2005); so, the three QTL for TSS identified in this study may be used for the breeding of high yield wheat varieties. Furthermore, the results revealed that SHW-L1 contributed positively to all the three major loci. Hence, future breeding programs can use the QTL-associated markers to fully exploit the genetic potential of QTL in increasing SHW-L1production.

A total of $23 \mathrm{~A}$. thaliana gene homologs were predicted in the three QTL intervals. The results of GO annotation suggest that seven candidate genes deserve our attention; these are: LECRK42, AT2G34930, PME21, COBL7, PIP5K4 (located in the QTSS.sicau-2D.2 intervals), CRK8 (located in the QTSS.sicau-2D.3 intervals), and RPPL1 (located in the QTSS.sicau-2D.1 intervals). Among them, LECRK42, PME21, and PIP5K4 play critical roles in pol- len and pollen tube development (Sousa et al., 2008; Wan et al., 2008; Oo et al., 2014); AT2G34930 encodes cell wall proteins in the apoplastic fluids of rosettes (Boudart et al., 2005); and COBL7 influences the development and function of the gynoecium (Scutt et al., 2003). Pollen and flower development is closely related to flowering time, and flowering time genes affect ear differentiation, including TSS (Jiang et al., 1982). Moreover, differentiation of TSS indicates a switch from vegetative to reproductive growth $(\mathrm{Li}, 1976)$. Interestingly, CRK8 is involved in reproductive signal transduction (Zhao et al., 2011), and $R P P L 1$, which interacts with GRF2, plays crucial roles in controlling growth and development in plants (Gökirmak et al., 2015; Ghorbel et al., 2017). For all these reasons, the seven candidate genes located in the three QTL intervals were considered to be closely related to TSS, which validates the accuracy of our results, provides reference for future map-based cloning experiments, and helps to better understand the genetic mechanism of spikelet growth and development in wheat.

\section{Conclusions}

In this study, we provided a strategy of identifying QTL by combining new molecular data with phenotypic data, and identified two novel QTL for TSS. A total of seven candidate genes associated with TSS were predicted. Overall, our data provides insight into the genetic basis of TSS, which might accelerate the development of highyielding wheat cultivars.

\section{Acknowledgments}

This study was financially supported by the National Natural Science Foundation of China (31560388 and 31771794), the outstanding Youth Foundation of the Department of Science and Technology of Sichuan Province (2016JQ0040), the Key Technology Research and Development Program of the Department of Science and Technology of Sichuan Province (2016NZ0057), and the International Science \& Technology Cooperation Program of the Bureau of Science and Technology of Chengdu China (No. 2015DFA306002015-GH03-00008-HZ).

\section{Conflict of interest}

The authors declare that they have no conflict of interest.

\section{Author contributions}

MD conducted data analysis and drafted the manuscript; FW performed the analysis of genotyping data and partially drafted the manuscript; WZ, JL, YuL and XY performed the phenotypic evaluation and helped with data analysis; HS and ZW performed part of the population genotyping; YW participated in the design of the study; YZ 
coordinated the study and helped to draft the manuscript; YaL designed and coordinated this study and revised the manuscript; All authors have read and approved the final manuscript.

\section{References}

Boudart G, Jamet E, Rossignol M, Lafitte C, Borderies G, Jauneau A, Esquerré-Tugayé MT and Pont-Lezica R (2005) Cell wall proteins in apoplastic fluids of Arabidopsis thaliana rosettes: Identification by mass spectrometry and bioinformatics. Proteomics 5:212-221.

Cai Q, Yuan Z, Chen M, Yin C, Luo Z, Zhao X, Liang W, Hu J and Zhang D (2014) Jasmonic acid regulates spikelet development in rice. Nat Commun 5, 3476-3476.

Cui F, Ding A, Li J, Zhao C, Wang L, Wang X, Qi X, Li X, Li G, Gao J and Wang H (2012) QTL detection of seven spikerelated traits and their genetic correlations in wheat using two related RIL populations. Euphytica 186:177-192.

Gökirmak T, Denison FC, Laughner BJ, Paul A-L and Ferl RJ (2015) Phosphomimetic mutation of a conserved serine residue in Arabidopsis thaliana 14-3-3w suggests a regulatory role of phosphorylation in dimerization and target interactions. Plant Physiol Biochem 97:296-303.

Ghorbel M, Cotelle V, Ebel C, Zaidi I, Ormancey M, Galaud J-P and Hanin M (2017). Regulation of the wheat MAP kinase phosphatase 1 by 14-3-3 proteins. Plant Sci 257:37-47.

Goddard M (1992) A mixed model for analyses of data on multiple genetic markers. Theor Appl Genet 83:878-886.

Godfray HC,Beddington JR,Crute IR,Haddad L,Lawrence D,Muir JF,Pretty J,Robinson S,Thomas SM andToulmin C (2010) Food security: The challenge of feeding 9 billion people. Science 327:812-818.

Jiang JY, Hua ZJ, An CH and Liu CF (1982) Comparative observation on the process of young spike differentiation of spring wheat varieties in different maturing stages. Ningxia J Agric Forestry Sci Technol 4,000. [in Chinese]

Kosambi DD (2016) The estimation of map distances from recombination values. In: Ramaswamy R (ed) D.D. Kosambi - Selected works in mathematics and statistics. Springer, New Dehli, pp 125-130.

Li W, Nelson J, Chu C, Shi L, Huang S and Liu D (2002) Chromosomal locations and genetic relationships of tiller and spike characters in wheat. Euphytica 125:357-366.

Li YQ (1976) Changes of polysaccharide during the differentiation of young spike in rice. J Wuhan Univ 2:60-67. [in Chinese]

Liu G, Jia L, Lu L, Qin D, Zhang J, Guan P, Ni Z, Yao Y, Sun Q and Peng H (2014) Mapping QTLs of yield-related traits using RIL population derived from common wheat and Tibetan semi-wild wheat. Theor Appl Genet 127:2415-2432.

Ma Z, Zhao D, Zhang C, Zhang Z, Xue S, Lin F, Kong Z, Tian D and Luo Q (2007) Molecular genetic analysis of five spikerelated traits in wheat using RIL and immortalized F2 populations. Mol Genet Genomics 277:31-42.

Mackay TFC (2001) The genetic architecture of quantitative traits Annu Rev Genet 35:303-339.

Mares D and Mrva K (2008) Genetic variation for quality traits in synthetic wheat germplasm. Austral J Agric Res 59:406412.
Marone D, Laidò G, Gadaleta A, Colasuonno P, Ficco DB, Giancaspro A, Giove S, Panio G, Russo MA, De Vita P et al. (2012) A high-density consensus map of A and B wheat genomes. Theor Appl Genet 125:1619-1638.

Nachit MM, Elouafi I, Pagnotta A, El Saleh A, Iacono E, Labhilili M, Asbati A, Azrak M, Hazzam H, Benscher D et al. (2001) Molecular linkage map for an intraspecific recombinant inbred population of durum wheat (Triticum turgidum L. var. durum). Theor Appl Genet 102:177-186.

Oo MM, Bae H-K, Nguyen TD, Moon S, Oh SA, Kim JH, Soh MS, Song JT, Jung KH and Park SK (2014) Evaluation of rice promoters conferring pollen-specific expression in a heterologous system, Arabidopsis. Plant Reprod 27:47-58.

Piepho H, Möhring J, Melchinger A and Büchse, A. (2008). BLUP for phenotypic selection in plant breeding and variety testing. Euphytica 161:209-228.

Quarrie S, Pekic Quarrie S, Radosevic R, Rancic D, Kaminska A, Barnes J, Leverington M, Ceoloni C and Dodig D (2006) Dissecting a wheat QTL for yield present in a range of environments: from the QTL to candidate genes. J Exp Bot 57:2627-2637.

Rajaram S (2001) Prospects and promise of wheat breeding in the 21st century. Euphytica 119:37-52.

Rawson H (1970) Spikelet number, its control and relation to yield per ear in wheat. Austral J Biol Sci 23:1-16.

Reynolds M (1996) Increasing yield potential in wheat: breaking the barriers. Proceedings of a workshop held in Ciudad Obregón, Sonora, Mexico: CIMMYT.

Roussel V, Leisova L, Exbrayat F, Stehno Z and Balfourier F (2005) SSR allelic diversity changes in 480 European bread wheat varieties released from 1840 to 2000 . Theor Appl Genet 111:162-170.

Scutt CP, Vinauger-Douard M, Fourquin C, Ailhas J, Kuno N, Uchida K, Gaude T, Furuya M and Dumas C (2003) The identification of candidate genes for a reverse genetic analysis of development and function in the Arabidopsis gynoecium. Plant Physiol 132:653-665.

Slafer G and Andrade FH (1993) Physiological attributes related to the generation of grain yield in bread wheat cultivars released at different eras. Field Crops Res 31:351-367.

Sourdille P, Cadalen T, Guyomarc'h H, Snape J, Perretant M, Charmet, G, Boeuf C and Bernard M (2003) An update of the Courtot Chinese Spring intervarietal molecular marker linkage map for the QTL detection of agronomic traits in wheat. Theor Appl Genet 106:530-538.

Sousa E, Kost B and Malhó R (2008) Arabidopsis phosphatidylinositol-4-monophosphate 5-kinase 4 regulates pollen tube growth and polarity by modulating membrane recycling. Plant Cell 20:3050-3064.

Van Ooijen JW (2006) JoinMap 4. Software for the calculation of genetic linkage maps in experimental populations. Kyazma BV, Wageningen, Netherlands, 33.

Van Ooijen JW (2009) MapQTL® 6, Software for the mapping of quantitative trait in experiment populations of diploid species. Kyazma BV, Wageningen.

Wan J, Patel A, Mathieu M, Kim S-Y, Xu D and Stacey G (2008) A lectin receptor-like kinase is required for pollen development in Arabidopsis. Plant Mol Biol 67:469-482.

Wurschum T (2012) Mapping QTL for agronomic traits in breeding populations. Theor Appl Genet 125:201-210. 
Yang J (2016) QTL mapping for pre-harvest sprouting resistance and molecular characterization of six grain germinationrelated genes in synthetic wheat. Triticeae Res Inst Sichuan Agric Univ 23-25. [in Chinese with English abstract]

Yu M, Chen GY, Zhang LQ, Liu YX, Liu DC, Wang JR, Pu ZE, Zhang L, Lan XJ, Wei YM et al. (2014). QTL mapping for important agronomic traits in synthetic hexaploid wheat derived from Aegiliops tauschii ssp. tauschii. J Integr Agric 13:1835-1844.

Zhai H, Feng Z, Li J, Liu X, Xiao S, Ni Z and Sun Q (2016) QTL analysis of spike morphological traits and plant height in winter wheat (Triticum aestivum L.) using a high-density SNP and SSR-based linkage map. Front Plant Sci 7:1617.

Zhang L, Liu D, Yan Z, Lan X, Zheng Y and Zhou Y (2004) Rapid changes of microsatellite flanking sequence in the allopolyploidization of new synthesized hexaploid wheat. Sci China Ser C: Life Sc 47:553-561.

Zhao X, Li YH and Lan XG (2011) Progress of cysteine-rich proteins in plants. Progr Modern Biomed 6,044. [in Chinese with English abstract]

Zhou Y, Conway B, Miller D, Marshall D, Cooper A, Murphy P, Chao S, Brown-Guedira G and Costa J (2017) Quantitative trait loci mapping for spike characteristics in hexaploid wheat. Plant Genome 10.

\section{Internet Resources}

International Wheat Genome Sequencing Consortium (IWGSC) database, https://urgi.ver-

sailles.inra.fr/blast_iwgsc/blast.php/ (April 21, 2018)

Ensembl Plants database, http://plants.ensembl.org/hmmer/index.html/ (April 21, 2018)

KOBAS 3.0 database, http://kobas.cbi.pku.edu.cn/ (April 21, 2018)

\section{Supplementary material}

The following online material is available for this article:

Table S1 - SNPs and candidate genes significantly associated with Total spikelet number per spike (TSS) QTL.

Associate Editor: Everaldo Gonçalves de Barros

License information: This is an open-access article distributed under the terms of the Creative Commons Attribution License (type CC-BY), which permits unrestricted use, distribution and reproduction in any medium, provided the original article is properly cited. 